\title{
Inference of super-exponential human population growth via efficient computation of the site frequency spectrum for generalized models
}

Feng $\mathrm{Gao}^{1, \uparrow}$ and Alon Keinan ${ }^{1, \uparrow}$

${ }^{1}$ Department of Biological Statistics and Computational Biology, Cornell University, Ithaca, NY 14853, USA

${ }^{\dagger}$ Corresponding author:

Alon Keinan, Department of Biological Statistics and Computational Biology, Cornell University, Ithaca, NY 14853. E-mail: ak735@cornell.edu.

Feng Gao, Department of Biological Statistics and Computational Biology, Cornell University, Ithaca, NY 14853. E-mail: fg237@cornell.edu 


\section{Abstract}

The site frequency spectrum (SFS) and other genetic summary statistics are at the heart of many population genetics studies. Previous studies have shown that human populations had undergone a recent epoch of fast growth in effective population size. These studies assumed that growth is exponential, and the ensuing models leave unexplained excess amount of extremely rare variants. This suggests that human populations might have experienced a recent growth with speed faster than exponential. Recent studies have introduced a generalized growth model where the growth speed can be faster or slower than exponential. However, only simulation approaches were available for obtaining summary statistics under such models. In this study, we provide expressions to accurately and efficiently evaluate the SFS and other summary statistics under generalized models, which we further implement in a publicly available software. Investigating the power to infer deviation of growth from being exponential, we observed that decent sample sizes facilitate accurate inference, e.g. a sample of 3000 individuals with the amount of data expected from exome sequencing allows observing and accurately estimating growth with speed deviating by $10 \%$ or more from that of exponential. Applying our inference framework to data from the NHLBI Exome Sequencing Project, we found that a model with a generalized growth epoch fits the observed SFS significantly better than the equivalent model with exponential growth $\left(p\right.$-value $\left.=3.85 \times 10^{-6}\right)$. The estimated growth speed significantly deviates from exponential ( $p$-value $<<10^{-12}$ ), with the best-fit estimate being of growth speed $12 \%$ faster than exponential.

Key words: coalescent, generalized models, population growth, human demographic history, software 


\section{Introduction}

Summary statistics of genetic variation play a vital role in population genetics studies, especially inference of demographic history. In particular, the site frequency spectrum (SFS) is a vital summary statistic of genetic data and is widely utilized by many demographic inference methods applied to humans and other organisms (Marth et al. 2004; Gutenkunst et al. 2009; Excoffier et al. 2013; Bhaskar et al. 2015; Liu and Fu 2015). Other demographic inference methods are based on sequentially Markov coalescent and utilize the most recent common ancestor $\left(T_{\mathrm{MRCA}}\right)$ and linkage disequilibrium patterns (Li and Durbin 2011; Harris and Nielsen 2013; MacLeod et al. 2013; Sheehan et al. 2013; Schiffels and Durbin 2014). As another example, several studies used the average pairwise difference between chromosomes (Hammer et al. 2008; Gottipati et al. 2011; Arbiza et al. 2014) and the SFS (Keinan et al. 2009) to study the relative effective population sizes between the human $\mathrm{X}$ chromosome and the autosomes. The wide application of such genetic summary statistics stresses the need for their fast and accurate computation under any model of demographic history, instead of their estimations via simulations or approximations (e.g., Hudson 2002; Gutenkunst et al. 2009).

Several recent demographic inference studies showed evidence that human populations have undergone a recent epoch of fast growth in effective population size (Gutenkunst et al. 2009; Coventry et al. 2010; Gravel et al. 2011; Nelson et al. 2012; Tennessen et al. 2012; Gazave et al. 2014). However, the above studies assumed that the growth is exponential. The observation of huge amount of extremely rare, previously unknown 
variants in several sequencing studies with large sample sizes (Nelson et al. 2012;

Tennessen et al. 2012; Fu et al. 2013) and the recent explosive growth in census population size suggests that human population might have experienced a recent superexponential growth, i.e. growth with speed faster than exponential (Coventry et al. 2010; Keinan and Clark 2012; Reppell et al. 2012, 2014). Hence, recent studies presented a new generalized growth model that extends the previous exponential growth model by allowing the growth speed to be exponential or faster/slower than exponential (Reppell et al. 2012, 2014). Modeling the recent growth by this richer family of models holds the promise of a better fit to human genetic data, and can also be applicable to other organisms that experienced growth. However, only simulation approaches are currently available for evaluating such a generalized growth demographic model (Reppell et al. 2012), which makes inference of demographic history computational intractable.

In this study, we first provide a set of explicit expressions for the computation of five summary statistics under a model of any number of epochs of generalized growth: (1) the time to the most recent common ancestor $\left(T_{\mathrm{MRCA}}\right),(2)$ the total number of segregating sites $(S),(3)$ the site frequency spectrum (SFS), (4) the average pairwise difference between chromosomes per site $(\pi)$, and (5) the burden of private mutations, $\operatorname{BPM}(\alpha)$, a summary statistic that has been recently introduced as sensitive to recent growth (Keinan and Clark 2012; Gao and Keinan 2014). We introduce a new software package that implements these expressions, EGGS (Efficient computation of Generalized models' Genetic summary Statistics), which facilitates fast and accurate generation of these summary statistics. We show that the numerically computed summary statistics match 
well with simulation results, and facilitates computation that is orders of magnitudes faster than that of simulations. By performing demographic inference on the SFS generated from simulated sequences, we then explore how many samples are needed for recovering parameters of a recent generalized growth epoch. Finally, we applied the software to investigate the nature of the recent growth in humans by inferring demographic models using the SFS of synonymous variants of 4,300 European individuals from the NHLBI Exome Sequencing Project (Tennessen et al. 2012; Fu et al. 2013).

\section{Methods and Materials}

\section{Generalized demographic models}

A demographic model $N(T)$ describes the changes of effective population size $N$ against time $T$. We consider time, measured in generations, as starting from 0 at present and increasing backward in time. Furthermore, we consider the families of demographic models that are constituted by any number of epochs of generalized growth, along the lines of (Bhaskar and Song 2014). More formally, there exists a minimal positive integer $L$ such that the demographic history of a population can be split into a model with $L+1$ epochs that are split by $L$ ordered different time points $T_{1}, T_{2}, \cdots, T_{L}\left(T_{0}=0<T_{1}<\right.$ $T_{2} \cdots<T_{L}<T_{L+1}=\infty$ ), with the $k^{\text {th }}$ epoch starting from $T_{k-1}$ and lasting through $T_{k}$ (thus the last epoch starts at time $T_{L}$ and continues into indefinite past, $T_{L+1}=\infty$ ). Such a history is considered as a generalized model if the population size in each epoch $N\left(T_{k-1} \leq T<T_{k}\right)$ can be described by the following differential equation regarding time $T$ (Reppell et al. 2012, 2014): 


$$
\frac{d N}{d T}=-r_{k} N^{b_{k}}
$$

where $k=1,2, \cdots, L+1$. Each epoch $(k=1,2, \cdots, L+1)$ can hence capture variety of changes patterns in effective population size. Specifically, if $r_{k}=0$, this epoch is of constant population size. When $r_{k} \neq 0, b_{k}$ controls the growth speed of this epoch: (1) if $b_{k}=1$, the epoch is of exponential growth $\left(r_{k}>0\right)$ or decline $\left(r_{k}<0\right)$ with rate $r_{k} ;(2)$ if $b_{k}>1$, the epoch is of faster-than-exponential (super-exponential) growth $\left(r_{k}>0\right.$ ) or decline $\left(r_{k}<0\right)$; (3) if $b_{k}<1$, the epoch is of slower-than-exponential (sub-exponential) growth $\left(r_{k}>0\right)$ or decline $\left(r_{k}<0\right)$. Linear growth or decline is also a special case of generalized models when $b_{k}=0$. An illustration of a generalized model with 5 epochs is provided in Figure 1, and more detailed explanation and illustrations in the Supporting Information.

The solution to Equation (1) has been derived (Reppell et al. 2012, 2014) to be

$$
N(T)=\left\{\begin{array}{ll}
\left(N_{k, \mathrm{i}}^{1-b_{k}}-r_{k}\left(T-T_{k-1}\right)\left(1-b_{k}\right)\right)^{\frac{1}{1-b_{k}}}, b_{k} \neq 1 \\
N_{k, \mathrm{i}} \mathrm{e}^{-r_{k}\left(T-T_{k-1}\right)}, & b_{k}=1
\end{array},\right.
$$

where $N_{k, \mathrm{i}}$ is the initial population size of the $k^{\text {th }}$ epoch. Each epoch $k$ is defined by 4 parameters: the starting population size $\left(N_{k, \mathrm{i}}\right)$, the ending population size $\left(N_{k, \mathrm{f}}\right)$, the duration of the epoch $\left(T_{k}-T_{k-1}\right)$ and the growth speed parameter $b_{k}$. The growth rate parameter, $r_{k}$ is an immediate function of these parameters, $r_{k}=r_{k}\left(N_{k, \mathrm{i}}, N_{k, \mathrm{f}}, b_{k}, T_{k}-\right.$ $T_{k-1}$ ), and hence does not need to be provided as an independent variable in defining the changes in effective population size during an epoch. Note that $N_{k+1, \mathrm{i}}$, the starting population size of the $(k+1)^{\text {th }}$ epoch is not necessarily the same as $N_{k, \mathrm{f}}$, the ending 
population size of the $k^{\text {th }}$ epoch. When it is not, it entails an instantaneous change in population size at time $T_{k}$.

\section{Explicit expressions for summary statistics of demographic models under arbitrary population size functions}

Previous studies showed that under Kingman's coalescent, given a demographic model $N(T)$, the expected time of the most recent common ancestor $\mathbb{E}\left[T_{\mathrm{MRCA}}^{p}\right]$ can be calculated by (Polanski et al. 2003)

$$
\mathbb{E}\left[T_{\mathrm{MRCA}}^{p}\right]=\sum_{j=2}^{p} A_{j}^{p} \psi_{j}
$$

where the superscript $p$ is the number of chromosomes (twice the diploid sample size), $\psi_{j}$ is the expected time of the first coalescent event when there are $j$ chromosomes at present, which is given by

$$
\psi_{j}=\int_{0}^{\infty} T \frac{\left(\begin{array}{l}
j \\
2
\end{array}\right)}{N(T)} \mathrm{e}^{-\int_{0}^{T} \frac{\left(\begin{array}{l}
j \\
2
\end{array}\right) d \sigma}{N(\sigma)}} d T=\int_{0}^{\infty} \mathrm{e}^{-\left(\begin{array}{l}
j \\
2
\end{array}\right) \Lambda(T)} d T
$$

where $\Lambda(T)=\int_{0}^{T} \frac{d \sigma}{N(\sigma)}$, and $A_{j}^{p}$ are constants (Tavare 1984; Takahata and Nei 1985;

Polanski et al. 2003) provided in Supporting Information.

The expected full relative SFS $\mathbb{E}\left[\xi^{p}\right]=\left(\mathbb{E}\left[\xi_{1}^{p}\right], \mathbb{E}\left[\xi_{2}^{p}\right], \ldots, \mathbb{E}\left[\xi_{p-1}^{p}\right]\right)$ can be computed by the following set of equations (Polanski and Kimmel 2003):

$$
\mathbb{E}\left[\xi_{i}^{p}\right]=\frac{\mathbb{E}\left[\ell_{i}^{p}\right]}{\mathbb{E}\left[\mathcal{L}^{p)}\right]} ; \mathbb{E}\left[\ell_{i}^{p}\right]=\sum_{j=2}^{p} W_{i, j}^{p} \psi_{j} ; \mathbb{E}\left[\mathcal{L}^{p}\right]=\sum_{j=2}^{p} V_{j}^{p} \psi_{j},
$$

where $\ell_{i}^{p}$ is the length of branches in the genealogy that have $i$ descendants and $\mathcal{L}^{p}=\sum_{i=1}^{p-1} \ell_{i}^{p}$ is the total length of all branches in the coalescent tree. The quantities $V_{j}^{p}$ 
and $W_{i, j}^{p}$ are constants (Polanski and Kimmel 2003), which we provide in the Supporting Information.

Naturally, the expected number of segregating sites is given by

$$
\mathbb{E}\left[S^{p}\right]=\mu_{0} L \mathbb{E}\left[\mathcal{L}^{p}\right]
$$

where $\mu_{0}$ is the mutation rate per site per generation and $L$ is the length of the locus under consideration. The average pairwise difference between chromosomes per site, $\mathbb{E}[\pi]$, can be calculated by

$$
\mathbb{E}[\pi]=2 \mu_{0} \mathbb{E}\left[T_{\mathrm{MRCA}}^{p=2}\right]
$$

The expected burden of private mutations $(\alpha)$ at sample size of $\left(\frac{p}{2}-1\right)$, defined as the proportion of heterozygous sites in a new diploid individual that are homozygous in the previous $\left(\frac{p}{2}-1\right)$ individuals, $\mathbb{E}\left[\alpha \frac{p}{2}-1\right]$ can be approximated by (Gao and Keinan 2014)

$$
\mathbb{E}\left[\alpha_{\frac{p}{2}-1}\right]=\frac{2}{p[1+\delta(1, p-1)]} \frac{\mathbb{E}\left[\ell_{1}^{p}\right]+\mathbb{E}\left[\ell_{p-1}^{p}\right]}{\mathbb{E}\left[\ell_{1}^{2}\right]}
$$

where $\delta(x, y)$ is Kronecker delta function.

The detailed description of the five summary statistics mentioned above is included in the Supporting Information.

\section{Evaluation of the expected time to the first coalescent event under generalized models}

The core of the evaluation lies in finding feasible and numerically stable functions for calculating $\psi_{j}$, the expected time of the first coalescent event when there are $j$ chromosomes at present. Previous studies give explicit expressions of $\psi_{j}$ for a demographic model constructed by exponential and constant-size epochs (Polanski and 
Kimmel 2003; Bhaskar et al. 2015). In this study, we give a comprehensive set of formulas for generalized models as introduced above. Define $\phi_{j}^{k}:=\int_{T_{k-1}}^{T_{k}} \mathrm{e}^{-\left(\begin{array}{l}j \\ 2\end{array}\right) \Lambda(T)} d T$, then $\psi_{j}=\sum_{k=1}^{L+1} \phi_{j}^{k}$, where $(L+1)$ is the total number of epochs. The quantity $\phi_{j}^{k}$ can be computed numerically by the following set of equations:

(1) If $r_{k}=0$ :

$$
\phi_{j}^{k}=\frac{N_{k, \mathrm{i}}}{\left(\begin{array}{l}
j \\
2
\end{array}\right)}\left[\mathrm{e}^{-\left(\begin{array}{l}
j \\
2
\end{array}\right) \Lambda\left(T_{k-1}\right)}-\mathrm{e}^{-\left(\begin{array}{l}
j \\
2
\end{array}\right) \Lambda\left(T_{k}\right)}\right]
$$

(2) If $b_{k}=0, r_{k} \neq 0$ :

$$
\phi_{j}^{k}=\left\{\begin{array}{c}
\frac{1}{\left(\begin{array}{c}
j \\
2
\end{array}\right)}\left[\mathrm{e}^{-\left(\begin{array}{c}
j \\
2
\end{array}\right) \Lambda\left(T_{k}\right)} N_{k, \mathrm{f}} \log N_{k, \mathrm{f}}-\mathrm{e}^{-\left(\begin{array}{c}
j \\
2
\end{array}\right) \Lambda\left(T_{k-1}\right)} N_{k, \mathrm{i}} \log N_{k, \mathrm{i}}\right], r_{k}+\left(\begin{array}{l}
j \\
2
\end{array}\right)=0 \\
\frac{1}{r_{k}+\left(\begin{array}{c}
j \\
2
\end{array}\right)}\left[\mathrm{e}^{-\left(\begin{array}{c}
j \\
2
\end{array}\right) \Lambda\left(T_{k-1}\right)} N_{k, \mathrm{i}}-\mathrm{e}^{-\left(\begin{array}{c}
j \\
2
\end{array}\right) \Lambda\left(T_{k}\right)} N_{k, \mathrm{f}}\right], r_{k}+\left(\begin{array}{l}
j \\
2
\end{array}\right) \neq 0
\end{array} ;\right.
$$

(3) If $b_{k}>0$ and $r_{k}>0$, or $b_{k}=1$ and $r_{k}<0$ :

$$
\begin{aligned}
\phi_{j}^{k}=\frac{1}{\left(\begin{array}{c}
j \\
2
\end{array}\right)}\left[N_{k, \mathrm{i}}\right. & \mathcal{U}\left(2-\frac{1}{b_{k}}, \frac{\left(\begin{array}{l}
j \\
2
\end{array}\right)}{b_{k} r_{k}} N_{k, \mathrm{i}}^{-b_{k}}\right) \mathrm{e}^{-\left(\begin{array}{l}
j \\
2
\end{array}\right) \Lambda\left(T_{k-1}\right)} \\
& \left.-N_{k, \mathrm{f}} u\left(2-\frac{1}{b_{k}}, \frac{\left(\begin{array}{l}
j \\
2
\end{array}\right)}{b_{k} r_{k}} N_{k, \mathrm{f}}^{-b_{k}}\right) \mathrm{e}^{-\left(\begin{array}{l}
j \\
2
\end{array}\right) \Lambda\left(T_{k}\right)}\right] ;
\end{aligned}
$$

(4) If $b_{k}<0$ and $r_{k}>0$ :

$$
\begin{aligned}
\phi_{j}^{k}=\frac{1}{\left(\begin{array}{l}
j \\
2
\end{array}\right)}\left[N_{k, \mathrm{f}} \mathcal{M}\left(2-\frac{1}{b_{k}}, \frac{\left(\begin{array}{c}
j \\
2
\end{array}\right)}{b_{k} r_{k}} N_{k, \mathrm{f}}^{-b_{k}}\right) \mathrm{e}^{-\left(\begin{array}{c}
j \\
2
\end{array}\right) \Lambda\left(T_{k}\right)}\right. \\
\left.\quad-N_{k, \mathrm{i}} \mathcal{M}\left(2-\frac{1}{b_{k}}, \frac{\left(\begin{array}{l}
j \\
2
\end{array}\right)}{b_{k} r_{k}} N_{k, \mathrm{i}}^{-b_{k}}\right) \mathrm{e}^{-\left(\begin{array}{c}
j \\
2
\end{array}\right) \Lambda\left(T_{k-1}\right)}\right] .
\end{aligned}
$$

The expressions of the $\Lambda(T)$ function are given in the Supporting Information. The function $\mathcal{U}(b, x):=x U(1, b, x)=x \int_{0}^{\infty} \mathrm{e}^{-x t}(1+t)^{b-2} d t$, where $U(a, b, x)$ is the confluent hypergeometric function of the second kind (Gradshteĭn et al. 2007). The 
function $\mathcal{M}(b, x):=\frac{x}{b-1} M(1, b, x)=x \int_{0}^{1} \mathrm{e}^{x t}(1-t)^{b-2} d t$, where $M(a, b, x)$ is the confluent hypergeometric function of the first kind (Gradshteĭn et al. 2007). The exponential growth or decline then becomes a special case of $\mathcal{U}(b, x)$ when $b=1$ and $x \neq 0$

$$
\mathcal{U}(1, x)=x \mathrm{e}^{x} \int_{1}^{\infty} \frac{\mathrm{e}^{-t}}{t} d t=x \mathrm{e}^{x} E_{1}(x)
$$

where $E_{1}(x)$ is the Exponential Integral (Gradshteĭn et al. 2007), which has been shown by previous studies (Polanski and Kimmel 2003; Bhaskar et al. 2015). We could not find feasible and numerically stable closed-form formulas for $\phi_{j}^{k}$ when the population size decreases forward in time in a manner that is not linear or exponential (i.e. $r_{k}<0$ and $\left.b_{k} \notin\{0,1\}\right)$. In these scenarios, we used Gauss-Legendre quadrature (Kahaner et al. 1988) for efficient numerical evaluation of relevant functions (see Supporting Information for a detailed description).

\section{Software implementation}

The above expressions are implemented in a software package, EGGS (Efficient computation of Generalized models' Genetic summary Statistics). The software and source code are publicly available from our website (http://keinanlab.cb.bscb.cornell.edu). Compiled versions for Linux and MAC OS are available. Source code is in $\mathrm{C}++$, with no external libraries needed for compilation. Additional information of implementation is included in the Supporting Information and in the manual that accompanies the software online. 


\section{Demographic models assumed in this study}

The demographic models used in this study are based on the inferred European history presented by Gazave et al. 2014 (Figure 2, in black), which contains two bottlenecks (Keinan et al. 2007) and a recent exponential growth epoch. Specifically, Gazave et al. 2014 model has a constant effective population size of 10,000 (diploid) individuals before 4,720 generations ago, and goes through the ancient bottleneck between 4,720 generations ago and 4,620 generations ago with a population size of 189 . The population size then recovers to 10,000 diploids until 720 generations ago, from which time the recent bottleneck starts with a size of 549. At 620 generations ago, the population size recovers to 5,633 individuals. The recent growth epoch starts from 140.8 generations ago and lead to population size of 654,000 at present. The parameters of the original recent growth epoch were varied to incorporate generalized growth effects.

\section{Optimization method of demographic inference based on the site frequency}

\section{Spectrum}

Demographic inference in this study was based on the observed allele frequency counts from the simulated or real dataset. To determine the fitness of a model to the observed data, we calculated the composite log likelihood by

$$
\mathbb{L}(\Theta)=\log \mathbb{P}(\boldsymbol{C} \mid \Theta)=\mathcal{C} \cdot \log \mathbb{E}(\boldsymbol{\eta} \mid \Theta),
$$

where $\mathcal{C}$ is a vector of the observed folded allele frequency counts and $\mathbb{E}(\boldsymbol{\eta} \mid \Theta)$ is the computed folded SFS under demographic model $\Theta$. More detailed description can be found in the Supporting Information. 
To search for the maximum likelihood point over the parameter space, we applied the

ECM method (Meng and Rubin 1993; Excoffier et al. 2013). 100 ECM cycles were performed for each run of inference. We obtained $95 \%$ confidence intervals of parameter estimates via block bootstrapping of the data 200 times.

\section{Processing of NHLBI Exome Sequencing Project data for demographic history inference}

The NHLBI Exome Sequencing Project (ESP) data (Tennessen et al. 2012; Fu et al. 2013) contains deep sequencing of 4,300 individuals of European ancestry. An important feature of these data is the high sequencing coverage, which allows to capture accurately very rare variants, which constitutes the part of the SFS that is most enriched for information on recent population growth (Keinan and Clark 2012; Tennessen et al. 2012; Gao and Keinan 2014). To reduce the effect of selection as much as possible while keeping sufficient amount of data, we chose to use the SFS calculated from synonymous single nucleotide variants (SNVs) only, as previously performed by Tennessen et al. 2012. To further improve the quality of the data, we filtered SNVs with average read depth less than or equal to 20 , or with successful genotype counts less than 7,740 (90\%), and subsampled the remaining 233,134 SNVs to 7,740 alleles, which is equivalent to 3,870 diploid individuals (Supporting Information). 


\section{Results}

\section{Comparison with simulated results by FTEC}

To validate that the expressions provided in the Methods and Materials section can correctly compute the summary statistics under generalized growth models, we compared the summary statistics calculated by our software EGGS to those simulated by the software FTEC (a coalescent simulator for modeling faster than exponential growth) (Reppell et al. 2012) under the demographic models shown in Figure 2(A). This model is the model of European history inferred in Gazave et al. 2014, except that we varied the growth speed parameter $b$ (Equation 1), which corresponds to 1 in this model (exponential growth), to also be 0.5 (corresponding to sub-exponential growth), and 1.5 (corresponding to super-exponential growth). The sample size is fixed at 1,000 diploid individuals (2,000 chromosomes). For FTEC simulation, we used mutation rate of $1.2 \times 10^{-8}$ per base pair per generation (e.g., Kong et al. 2012) and simulated 200,000 independent loci, each of 1,000 base pairs.

The comparison of the SFS, $S$ (across all 200,000 loci), $\pi$ and $\alpha$ numerically computed by EGGS to that simulated by FTEC is shown in Figure 2(B)-(E). For each demographic model illustrated in Figure 2(A), the values for all summary statistics from the numerical computation by EGGS are practically identical to those from the simulation results by FTEC. However, our software EGGS exhibits a huge speed improvement over FTEC. For each model considered in Figure 2(A), EGGS takes less than a second to generate the results, while it takes about 5 hours for FTEC to simulate the sequences, due to the large number of independent loci required for accurate estimation (performed in Ubuntu 
system with Intel Xeon CPU @ 2.67GHz). For instance, when 2,000 independent loci are simulated, which still takes about 3 minutes, the summary statistics deviate considerably from the accurate results (Figure S2 and Table S1). Furthermore, our software works well over a wide range of values of the growth parameter $b$, even when $b$ is 0 (corresponding to linear growth or decline) or $b<0$ (Figure S3), conditions that are not handled by FTEC. We note, however, that as a simulation program FTEC provides the full sequences as output and can have a wider range of applications than facilitated by the SFS and other summary statistics that EGGS calculates.

\section{Evaluating inference of generalized growth based from the site frequency spectrum}

We next set out to test the accuracy (as a function of sample size) of inferring parameters in models with generalized growth from the SFS. A recent study has shown that, in theory, an underlying generalized growth demographic model can be uniquely identified by the ideal, perfect expected SFS of a very small sample size generated from that model (34 haploid sequences for the models shown in Figure 2(A)) (Bhaskar and Song 2014). However, the SFS is estimated in practice from limited amount of data from each individual (even in the case of whole-genome sequencing) and, hence, the estimated SFS will fluctuate around the expected values, which limits its accuracy for inference (Terhorst and Song 2015). We aim to test such inference in practice and determine the power of generalized growth detection and the sample size needed for accurately recovering the growth parameter and other parameters of the demographic model. To be comparable with many practical applications, we considered sequence length that is about equivalent to that obtained from whole exome sequencing (Supporting Information). 
We performed inference on the SFS calculated from simulated sequences generated by FTEC. We simulated a demographic model with the same initial epochs as the model illustrated in Figure 2(A). Starting 620 generations ago, the simulated model included a constant population size of 10,000 until 200 generations ago, when the population starts a generalized growth epoch till the present. The generalized growth epoch starts with a population size of 10,000 that grows to an extant effective population size of 1 million individuals, with the growth speed parameter $b$ taking either of the following values: 0.4 , $0.7,0.9,1.0,1.1,1.3$ and 1.6. We chose these values to represent a range of superexponential and sub-exponential growth, with emphasis on values around the exponential rate $(b=1.0)$ in order to test the detection power of generalized growth when the growth speed deviates slightly from exponential. We varied the sample size (number of diploid individuals sampled at present) to be 1,000, 2,000, 3,000, 5,000 and 10,000 (Supporting Information). The first 15 entries of the site frequency spectra for these simulated scenarios are shown in Figure S4. From each set of simulations, we then infer four parameters of the recent growth epoch, which can uniquely determine the epoch: 1) the growth speed parameter $b ; 2$ ) the initial population size before growth $N_{\mathrm{i}} ; 3$ ) the ending population size after growth $N_{\mathrm{f}}$; and 4) the onset time of growth $T$, which is equivalent to the growth duration since the simulated epoch ends at present.

As sample size increases, the accuracy of the point estimates generally improves and the confidence interval narrows (Figure 3). Specifically, when the SFS of only 1,000 diploids is used for inference, the inference performs badly for all parameters, defined by large 
confidence intervals (Figure 3). However, the confidence interval always includes the true simulated value. A sample size of 2,000 already exhibits acceptable performance except when the growth speed becomes large $(b=1.3$ and 1.6). Yet larger sample sizes of 5,000 and 10,000 are sufficient for inferring all parameters with very tight confidence intervals. For such sample size, the inference even significantly distinguishes between growth speeds $(b=0.9$ and $b=1.1)$ that are close to exponential $(b=1.0)$ from that of an exponential, thereby concluding that a sub-exponential (0.9) or super-exponential (1.1) growth has taken place. These observations suggest that a sample size of at least 3,000 diploid individuals might be needed for inferring the parameters associated with the simulated recent generalized growth epoch, which is motivated by previous models of European demographic history. It remains to be explored how accurate are the estimates, and how their accuracy improves with sample size, across a more diverse set of models.

\section{European demographic history inference}

We next performed demographic inference on the NHLBI Exome Sequencing Project (ESP) data (Tennessen et al. 2012; Fu et al. 2013). We applied our inference to these data while considering and comparing two models. Both models assume the ancient epochs before 620 generations ago to be the same as those in the model illustrated in Figure 2(A). We infer the parameters only for the most recent epoch, which is one of generalized growth in one model, while is limited to exponential growth in the other. The parameters for inference are: (1) population size before growth $\left(N_{\mathrm{i}}\right),(2)$ population size after growth $\left(N_{\mathrm{f}}\right),(3)$ growth onset time $(T)$, which is equivalent to the duration of growth, for both models; and (4) only for the generalized growth model, the growth speed parameter $(b)$, 
which is fixed at $b=1$ for the exponential growth model. The point estimates and $95 \%$ confidence intervals are shown in Table 2 and the best-fit demographic models are illustrated in Figure 4.

Although our exponential growth model assumed a different ancient history before the recent growth epoch than that assumed in Tennessen et al. 2012, using ESP data and assuming exponential growth, the inferred growth epoch is generally consistent with that obtained in their study (Figure 4 and Table 1). Our study infers recent growth that started 198 (95\% CI: 195-202) generations ago from effective population size of $\sim 13,100$ $(12,600-13,600)$ and continued at a rate of $2.2 \%(2.15 \%-2.26 \%)$ per generation (Table 2$)$, while Tennessen et al. 2012 estimated that recent growth had an initial population size of $\sim 9,500$ individuals, a duration of 204 generations and a growth rate of $2.0 \%$ per generation.

The inferred generalized growth model fits the data significantly better than that with exponential growth $\left(p\right.$-value $=3.85 \times 10^{-6}$ by $\chi^{2}$ likelihood-ratio test with 1 degree of freedom). It estimates that growth started 213 (206-220) generations ago from an effective population size of $\sim 12,400(11,800-13,000)$, both values consistent with those estimated in the exponential growth model. The extant effective population size following growth is estimated to be $1.26(1.16-1.37)$ million. The inferred growth speed parameter $b=1.12(1.07-1.15)$ is significantly larger than exponential speed of $b=1(p$ value $<<10^{-12}$ using one-tailed $z$-test), which is the main difference between the two models. $b=1.12$ implies a growth rate acceleration pattern (Supporting Information) that 
is super-exponential at 12\% faster than exponential through the epoch (Figure 4): the super-exponential growth is relatively slow around the onset time, and it keeps accelerating as time approaches present.

Motivated by these results, we considered a third model with two recent exponential growth epochs, which still assumes the ancient epochs before 620 generations ago to be the same as those in the model illustrated in Figure 2(A). Five parameters are inferred (Table S2), with the first phase of growth estimated to have started 219 (95-334) generations ago with a population size of $12,200(11,700-13,200)$. This phase of growth lasts until 135 (25-157) generations ago and leads to a population size of 47,100 (30,200$540,900)$. The population size after the recent phase of growth is $1.12(1.07-2.09)$ million. This model provides a significantly better fit than the model with a single exponential growth $\left(p\right.$-value $=5.55 \times 10^{-6}$ by $\chi^{2}$ likelihood-ratio test with 2 degrees of freedom $)$, but is a worse model than the generalized growth model (based on Bayesian Information Criterion, $\left.\mathrm{BIC}_{\mathrm{two} \text {-epoch-exponential }}-\mathrm{BIC}_{\text {generalized }}=6.1\right)$. However, this model exhibits some of the same accelerating pattern as the generalized growth model, as ascertained by the growth rate of the most recent exponential epoch being $2.4 \%(2.3 \%-5.2 \%)$, larger than that of the first exponential epoch, $1.6 \%(1.3 \%-2.1 \%)$. This acceleration pattern shown in both the generalized model and the model with two exponential epochs is consistent with evidence of growth in European census population size that has greatly accelerated in Modern Era (Keinan and Clark 2012). 


\section{Discussion}

In this study, we provide the mathematical derivation and a software that can efficiently compute the expected values of five genetic data summary statistics given a generalized demographic model by evaluating the derived explicit expressions. These summary statistics include the time to the most recent common ancestor $\left(T_{\mathrm{MRCA}}\right)$, the total number of segregating sites $(S)$, the site frequency spectrum (SFS), the average pairwise difference between chromosomes per site $(\pi)$ and the burden of private mutation $(\alpha)$. The fast and accurate generation of these summary statistics is not limited to human demographic inference and will facilitate a variety of population genetic studies in humans and other organisms.

It is also possible that other families of growth models may fit the pattern of human population size history. For instance, previous studies also considered the algebraicgrowth model in the form of $N(T)=T^{\gamma}$ (Eldon et al. 2015). In reality, however, not all demographic models have numerically stable closed-form expressions for the expected time of the first coalescent event $\left(\psi_{j}\right)$. In these cases, fast and accurate numerical integration methods, such as Gauss-Legendre quadrature used in this work, can be applied to evaluate $\psi_{j}$. This technique holds the promise of efficiently generating the population genetic summary statistics under arbitrary population size functions.

In future studies, it will be valuable to incorporate gradient-based optimization techniques for the fast inference of demographic models containing generalized growth epochs, e.g. by extending the work of Bhaskar et al. 2015. Beyond the direct application for 
demographic inference and other population genetics analyses, several problems under the framework of $\Lambda$-coalescent (Birkner et al. 2013) can also be explored by using the results from this study. If the sample size is large and multi-merger and simultaneousmerger events are permitted, it is shown that under exponential growth models, the expected number of rare variants differs greatly from that derived from Kingman's coalescent (Bhaskar et al. 2014). This can be extended to the case of generalized growth. For instance, when the growth speed is faster than exponential, the SFS of the $\Lambda$ coalescent is expected to differ even more greatly from the of Kingman's coalescent. Similarly, following the recent work of Eldon et al. 2015, a natural question to ask is in what cases can the SFS distinguish between generalized growth models and multi-merger coalescent events.

By applying inference of generalized growth on the SFS generated from the synonymous variants of 4,300 individuals of NHLBI ESP dataset (Tennessen et al. 2012; Fu et al. 2014), we find that generalized growth model shows a better fit to the observed data than the exponential growth model that has been used by all previous demographic modeling studies $\left(p\right.$-value $\left.=3.85 \times 10^{-6}\right)$. We also find that the European population experiences a recent growth in population size with speed modestly faster than exponential $(b=1.12, p$ value $<<10^{-12}$ for difference from $b=1$ ). This result is consistent with previous speculations that human population might have undergone a recent accelerated growth epoch based on the observation of very rare, previously unknown variants in several sequencing studies with large sample sizes (Nelson et al. 2012; Tennessen et al. 2012; Fu et al. 2013). It is also in line with the super-exponential growth in census population size 
during that time (Keinan and Clark 2012). With the increasing availability of high-quality sequencing data with large sample sizes (Tennessen et al. 2012; Fu et al. 2013; Taylor et al. 2015), more refined and reasonable demographic models can be estimated for different human populations by the application of generalized growth models.

\section{Acknowledgments}

The authors would like to thank Leo Arbiza for helpful comments, Yun S. Song for insightful comments on earlier versions of this manuscript, and Arjun Biddanda for his careful editing of the software manual. This work was supported by National Institutes of Health grants R01GM108805 and R01HG006849, an award from The Ellison Medical Foundation, and an award from The Edward Mallinckrodt, Jr. Foundation. Feng Gao is a Howard Hughes Medical Institute International Student Research fellow.

\section{References}

Arbiza, L., S. Gottipati, A. Siepel, and A. Keinan, 2014 Contrasting X-linked and autosomal diversity across 14 human populations. Am J Hum Genet 94 (6):827844.

Bhaskar, A., A.G. Clark, and Y.S. Song, 2014 Distortion of genealogical properties when the sample is very large. Proc Natl Acad Sci US A 111 (6):2385-2390.

Bhaskar, A., and Y.S. Song, 2014 Descartes' Rule of Signs and the Identifiability of Population Demographic Models from Genomic Variation Data. Annals of Statistics 42 (6):2469-2493. 
Bhaskar, A., Y.X. Wang, and Y.S. Song, 2015 Efficient inference of population size histories and locus-specific mutation rates from large-sample genomic variation data. Genome Res 25 (2):268-279.

Birkner, M., J. Blath, and B. Eldon, 2013 Statistical properties of the site-frequency spectrum associated with lambda-coalescents. Genetics 195 (3):1037-1053.

Coventry, A., L.M. Bull-Otterson, X. Liu, A.G. Clark, T.J. Maxwell et al., 2010 Deep resequencing reveals excess rare recent variants consistent with explosive population growth. Nat Commun 1:131.

Eldon, B., M. Birkner, J. Blath, and F. Freund, 2015 Can the site-frequency spectrum distinguish exponential population growth from multiple-merger coalescents? Genetics 199 (3):841-856.

Excoffier, L., I. Dupanloup, E. Huerta-Sanchez, V.C. Sousa, and M. Foll, 2013 Robust demographic inference from genomic and SNP data. PLoS Genet 9 (10):e1003905.

Fu, W., R.M. Gittelman, M.J. Bamshad, and J.M. Akey, 2014 Characteristics of neutral and deleterious protein-coding variation among individuals and populations. Am J Hum Genet 95 (4):421-436.

Fu, W., T.D. O'Connor, G. Jun, H.M. Kang, G. Abecasis et al., 2013 Analysis of 6,515 exomes reveals the recent origin of most human protein-coding variants. Nature 493 (7431):216-220.

Gao, F., and A. Keinan, 2014 High burden of private mutations due to explosive human population growth and purifying selection. BMC Genomics 15 Suppl 4:S3. 
Gazave, E., L. Ma, D. Chang, A. Coventry, F. Gao et al., 2014 Neutral genomic regions refine models of recent rapid human population growth. Proc Natl Acad Sci U S A $111(2): 757-762$.

Gottipati, S., L. Arbiza, A. Siepel, A.G. Clark, and A. Keinan, 2011 Analyses of X-linked and autosomal genetic variation in population-scale whole genome sequencing. Nat Genet 43 (8):741-743.

Gradshteĭn, I.S., I.M. Ryzhik, and A. Jeffrey, 2007 Table of integrals, series, and products. Amsterdam ; Boston: Academic Press.

Gravel, S., B.M. Henn, R.N. Gutenkunst, A.R. Indap, G.T. Marth et al., 2011 Demographic history and rare allele sharing among human populations. Proc Natl Acad Sci U S A 108 (29):11983-11988.

Gutenkunst, R.N., R.D. Hernandez, S.H. Williamson, and C.D. Bustamante, 2009 Inferring the joint demographic history of multiple populations from multidimensional SNP frequency data. PLoS Genet 5 (10):e1000695.

Hammer, M.F., F.L. Mendez, M.P. Cox, A.E. Woerner, and J.D. Wall, 2008 Sex-biased evolutionary forces shape genomic patterns of human diversity. PLoS Genet 4 (9):e1000202.

Harris, K., and R. Nielsen, 2013 Inferring demographic history from a spectrum of shared haplotype lengths. PLoS Genet 9 (6):e1003521.

Hudson, R.R., 2002 Generating samples under a Wright-Fisher neutral model of genetic variation. Bioinformatics 18 (2):337-338.

Kahaner, D., C.B. Moler, S. Nash, and G.E. Forsythe, 1988 Numerical methods and software. Englewood Cliffs, N.J.: Prentice Hall. 
Keinan, A., and A.G. Clark, 2012 Recent explosive human population growth has resulted in an excess of rare genetic variants. Science 336 (6082):740-743.

Keinan, A., J.C. Mullikin, N. Patterson, and D. Reich, 2007 Measurement of the human allele frequency spectrum demonstrates greater genetic drift in East Asians than in Europeans. Nat Genet 39 (10):1251-1255.

Keinan, A., J.C. Mullikin, N. Patterson, and D. Reich, 2009 Accelerated genetic drift on chromosome X during the human dispersal out of Africa. Nat Genet 41 (1):66-70.

Kong, A., M.L. Frigge, G. Masson, S. Besenbacher, P. Sulem et al., 2012 Rate of de novo mutations and the importance of father's age to disease risk. Nature 488 (7412):471-475.

Li, H., and R. Durbin, 2011 Inference of human population history from individual whole-genome sequences. Nature 475 (7357):493-496.

Liu, X., and Y.X. Fu, 2015 Exploring population size changes using SNP frequency spectra. Nat Genet 47 (5):555-559.

MacLeod, I.M., D.M. Larkin, H.A. Lewin, B.J. Hayes, and M.E. Goddard, 2013 Inferring demography from runs of homozygosity in whole-genome sequence, with correction for sequence errors. Mol Biol Evol 30 (9):2209-2223.

Marth, G.T., E. Czabarka, J. Murvai, and S.T. Sherry, 2004 The allele frequency spectrum in genome-wide human variation data reveals signals of differential demographic history in three large world populations. Genetics 166 (1):351-372.

Meng, X.L., and D.B. Rubin, 1993 Maximum-Likelihood-Estimation Via the Ecm Algorithm - a General Framework. Biometrika 80 (2):267-278. 
Nelson, M.R., D. Wegmann, M.G. Ehm, D. Kessner, P. St Jean et al., 2012 An abundance of rare functional variants in 202 drug target genes sequenced in 14,002 people. Science 337 (6090):100-104.

Polanski, A., A. Bobrowski, and M. Kimmel, 2003 A note on distributions of times to coalescence, under time-dependent population size. Theoretical Population Biology 63 (1):33-40.

Polanski, A., and M. Kimmel, 2003 New explicit expressions for relative frequencies of single-nucleotide polymorphisms with application to statistical inference on population growth. Genetics 165 (1):427-436.

Reppell, M., M. Boehnke, and S. Zollner, 2012 FTEC: a coalescent simulator for modeling faster than exponential growth. Bioinformatics 28 (9):1282-1283.

Reppell, M., M. Boehnke, and S. Zollner, 2014 The impact of accelerating faster than exponential population growth on genetic variation. Genetics 196 (3):819-828.

Schiffels, S., and R. Durbin, 2014 Inferring human population size and separation history from multiple genome sequences. Nat Genet 46 (8):919-925.

Sheehan, S., K. Harris, and Y.S. Song, 2013 Estimating variable effective population sizes from multiple genomes: a sequentially markov conditional sampling distribution approach. Genetics 194 (3):647-662.

Takahata, N., and M. Nei, 1985 Gene Genealogy and Variance of Interpopulational Nucleotide Differences. Genetics 110 (2):325-344.

Tavare, S., 1984 Line-of-Descent and Genealogical Processes, and Their Applications in Population-Genetics Models. Theoretical Population Biology 26 (2):119-164. 
Taylor, P.N., E. Porcu, S. Chew, P.J. Campbell, M. Traglia et al., 2015 Whole-genome sequence-based analysis of thyroid function. Nat Commun 6:5681.

Tennessen, J.A., A.W. Bigham, T.D. O'Connor, W. Fu, E.E. Kenny et al., 2012 Evolution and functional impact of rare coding variation from deep sequencing of human exomes. Science 337 (6090):64-69.

Terhorst, J., and Y.S. Song, 2015 Fundamental limits on the accuracy of demographic inference based on the sample frequency spectrum. Proc Natl Acad Sci U S A 112 (25):7677-7682. 


\section{Figures and Tables}

Figure 1. Illustration of an example of a generalized demographic model as introduced in the first section of Methods and Materials. This model consists of 5 epochs (starting from present): (1) Faster-than-exponential $(b>1)$ growth (looking forward in time) from $N_{1, \mathrm{f}}$ to $N_{1, \mathrm{i}}$ between $T_{0}=0$ and $T_{1} ;$ (2) Linear decline (a special case of generalized growth, $b=0$ ) from $N_{2, \mathrm{f}}$ to $N_{2, \mathrm{i}}$ between $T_{1}$ and $T_{2} ;$ (3) Exponential growth (a special case of generalized growth, $b=1$ ) from $N_{3, \mathrm{f}}$ to $N_{3, \mathrm{i}}$ between $T_{2}$ and $T_{3}$; (4) Slower-thanexponential $(b<1)$ decline from $N_{4, \mathrm{f}}$ to $N_{4, \mathrm{i}}$ between $T_{3}$ and $T_{4} ;(5)$ Constant population size (a special case of generalized growth, $r=0$ ) at $N_{5, \mathrm{i}}=N_{5, \mathrm{f}}$ starting from $T_{4}$ and lasts indefinitely backward in time $\left(T_{5}=\infty\right)$. The ending population size of the previous epoch is not necessarily the beginning population size of the next epoch (e.g., $N_{2, \mathrm{f}} \neq N_{3, \mathrm{i}}$, $N_{4, \mathrm{f}} \neq N_{5, \mathrm{i}}$ ), corresponding to an instantaneous population size change at that time.

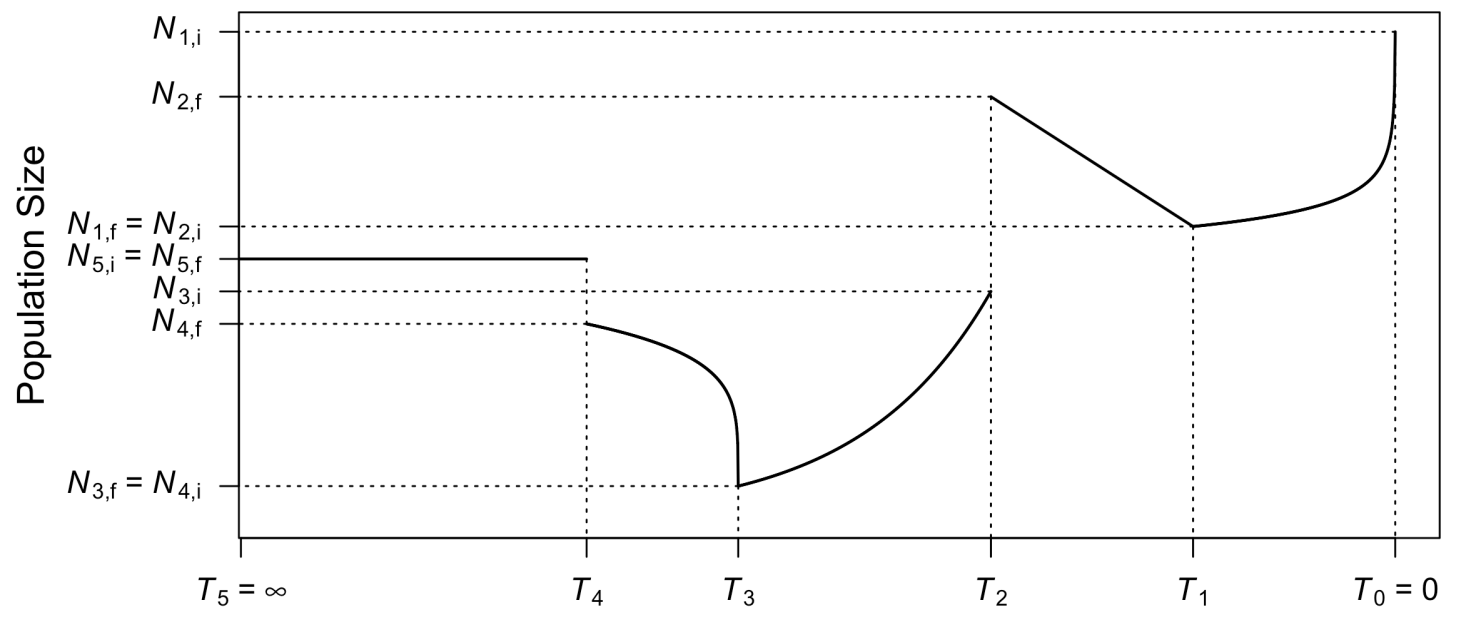

Time (Generations) 
Figure 2. (A) Demonstration of the demographic models considered for evaluating the accuracy of our calculations as implemented in EGGS (first section of Results). This twobottleneck model has the same population size and time throughout history as in the inferred European history in Gazave et al. 2014, with the exception that we varied the growth speed parameter of the recent growth epoch to be $b=0.5$ (sub-exponential, blue), $b=1.0$ (exponential as in Gazave et al. 2014, black) and $b=1.5$ (super-exponential, red). The $y$-axis shows effective population size of diploid individuals on a log-scale. (B)-(E) The comparison of the first 15 entries of the SFS (B), the total number of segregating sites $(S)$ across all 200,000 loci (1,000 bp-long each) (C), the expected pairwise difference between chromosomes per base pair (D) and the burden of private mutation $(\alpha)$ as the percentage of heterozygous variants in one individual that are monomorphic in the rest of the sample of 999 individuals (E) computed numerically in EGGS (dark bars) and simulated by FTEC (light bars) for the demographic models shown in (A): $b=0.5$, blue; $b=1.0$, black; $b=1.5$, red, with a sample size of 1,000 individuals $(2,000$ chromosomes $)$. The $y$-axis in (B) is on log scale. 

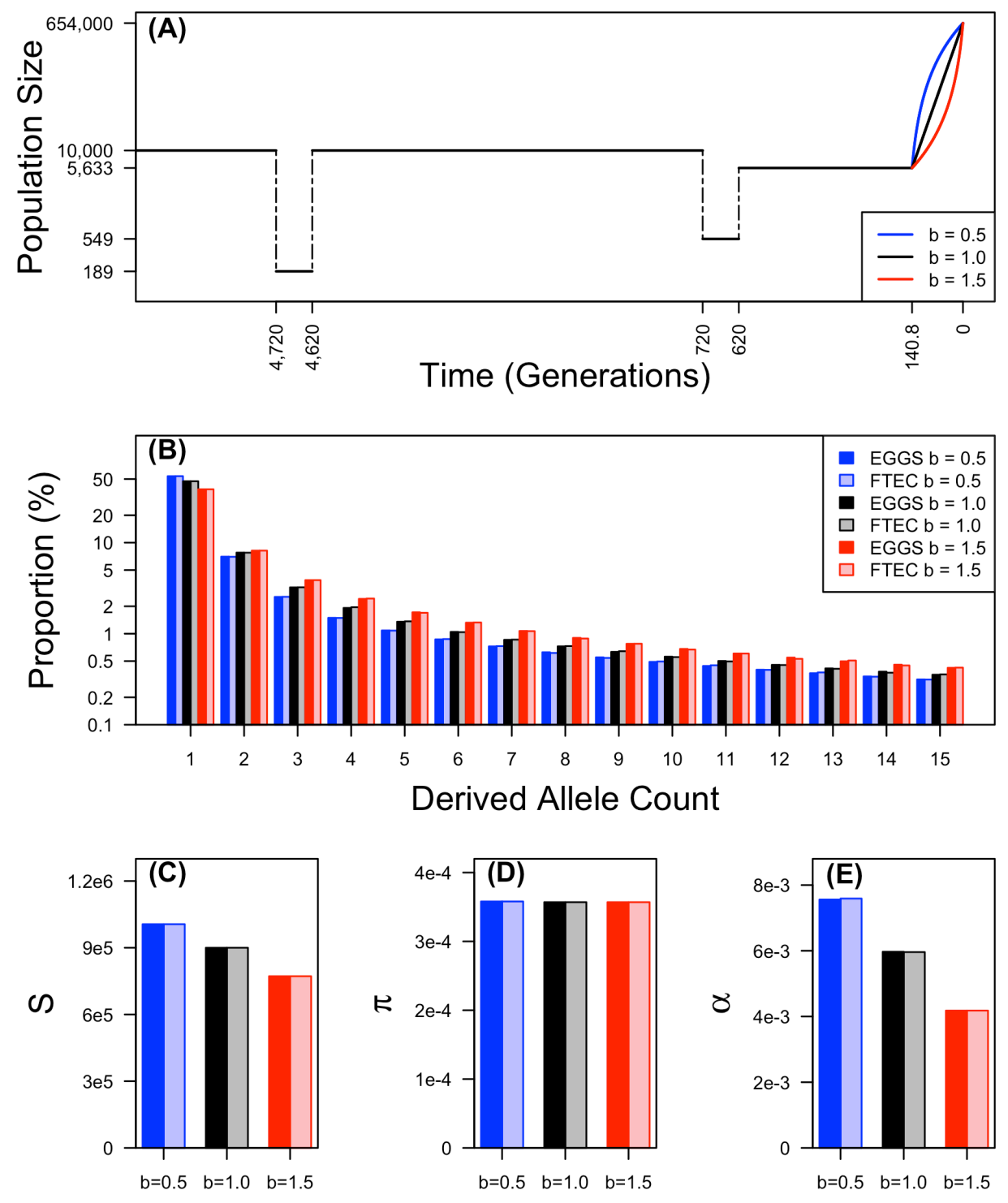
Figure 3. Inference results on simulated data with a recent growth epoch with the following parameters: Growth starts 200 generations before present from an effective population size of 10,000 and ends with an effective population size of 1 million at present. The growth speed parameter $b$ takes the following values in different simulations: $0.4,0.7,0.9,1.0,1.1,1.3$, and 1.6. Inference of these four parameters is based on the SFS estimated from a sample of individuals of one of five sizes $(1,000$, black; 2,000, red; 3,000, blue; 5,000, dark red; and 10,000, green). The point estimates with $95 \%$ confidence interval for these are grouped by the growth speed parameter $b$ (x-axis). The dashed purple lines show the true values of the simulated model. The results are shown in the following order: (A) the inferred growth speed parameter, (B) the inferred population size before growth, (C) the inferred population size after growth, (D) the inferred growth start time. The $y$-axis in $(\mathrm{C})$ is on log-scale. 
bioRxiv preprint doi: https://doi.org/10.1101/022574; this version posted July 15,2015 . The copyright holder for this preprint (which was not certified by peer review) is the author/funder, who has granted bioRxiv a license to display the preprint in perpetuity. It is made available under aCC-BY-NC-ND 4.0 International license.
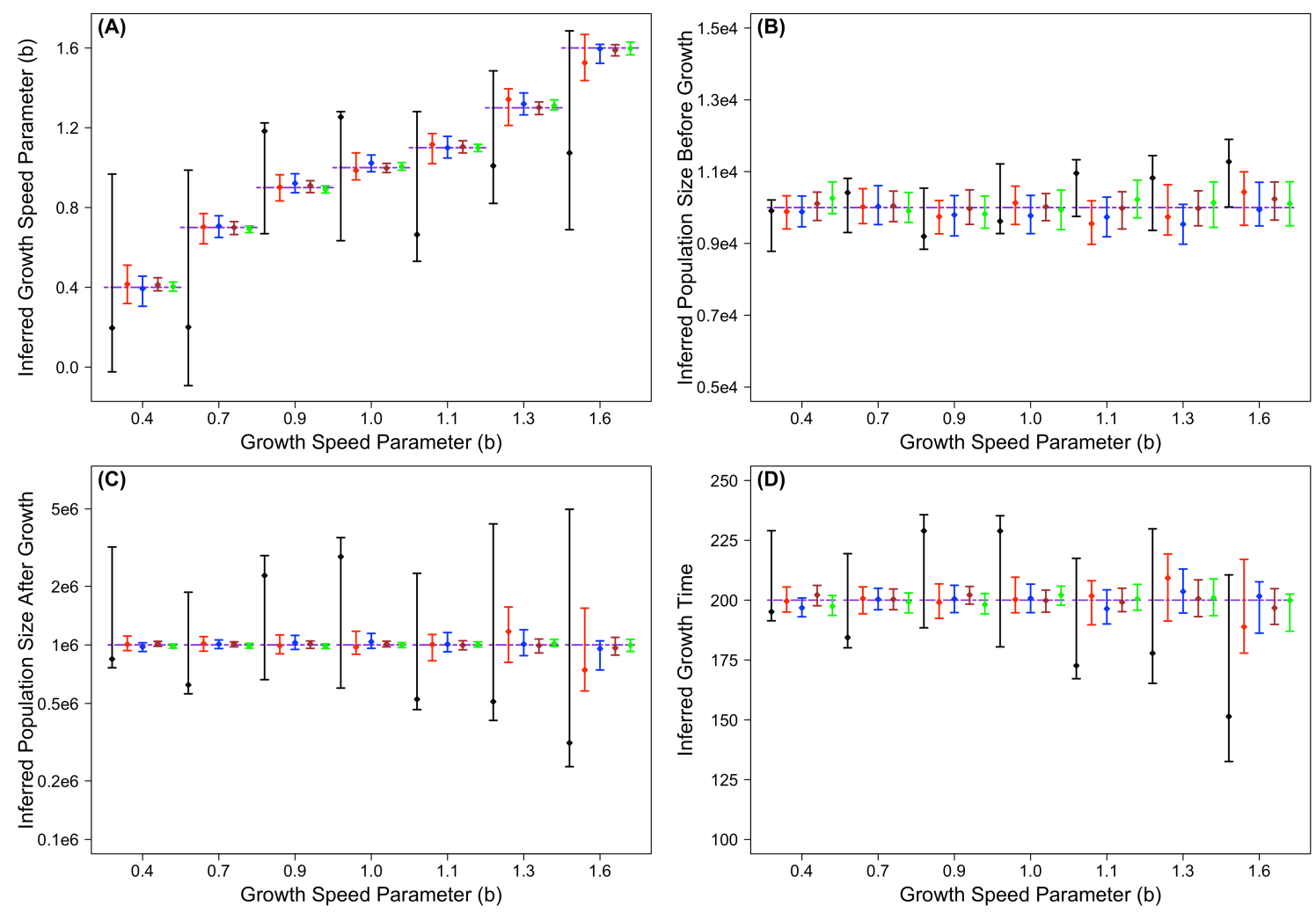
Figure 4. (A) Illustration of the effective population size (y-axis, on log scale) over time for the best-fit models inferred based on ESP data. Two models are shown: One restricted to recent growth being exponential (red) and one with a generalized recent growth epoch (black). Before 620 generations ago, the model is not inferred and all parameters are set to be the same as those shown in Figure 2(A). Solid lines show the effective population size over time of each of the inferred models, with dashed line indicating estimating parameter values on the $x$-axis or $y$-axis. Only the most recent 800 generations are shown to emphasize the difference between the two models. (B) A zoom-in to the most recent 200 generations of the inferred models to emphasize the acceleration pattern of the generalized growth model, with $y$-axis on normal scale.

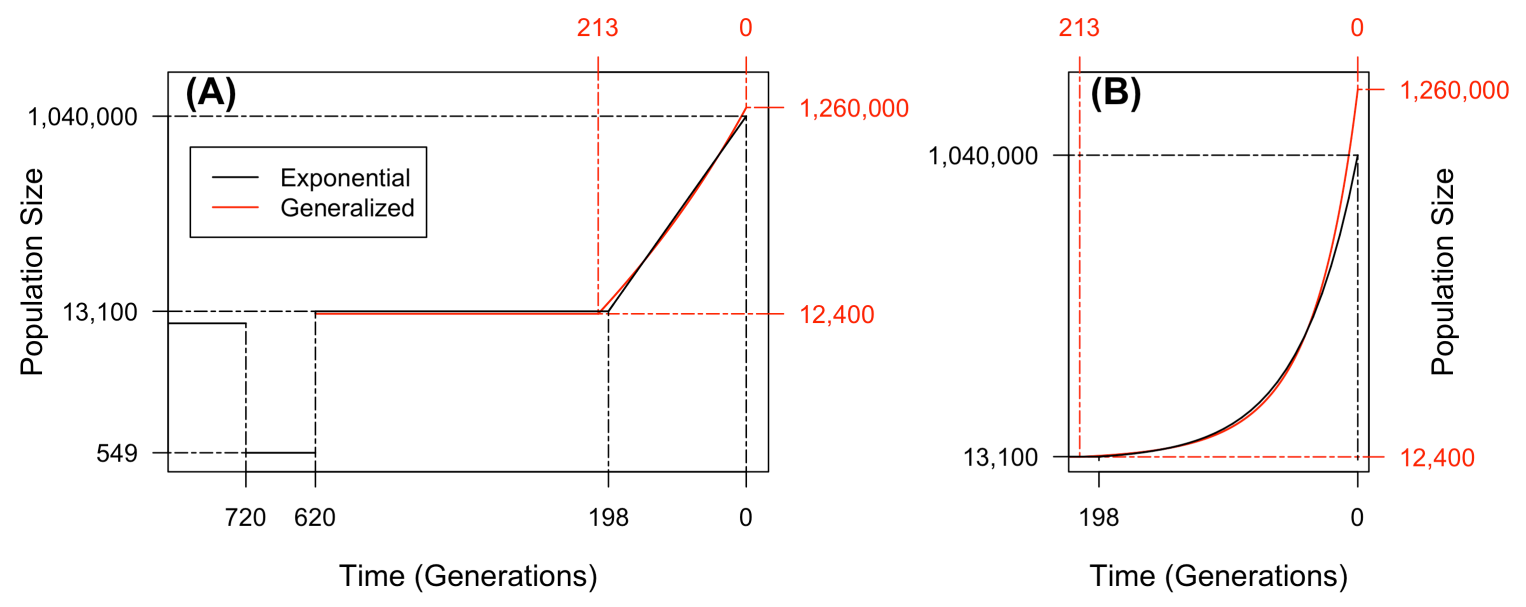


Table 1. Demographic inference results using ESP data for a model with a recent epoch of exponential growth and a model of recent epoch of generalized growth. Shown are point estimates and $95 \%$ confident intervals (in parenthesis) for the following parameters of the inferred epoch: population size before growth $\left(N_{\mathrm{i}}\right)$, population size after growth $\left(N_{\mathrm{f}}\right)$, time growth started, in generations $(T)$, and the growth speed parameter $(b)$, which is fixed at $b=1$ in the exponential growth case.

\begin{tabular}{|c|c|c|c|c|}
\hline & $N_{\mathrm{i}}\left(10^{4}\right)$ & $N_{\mathrm{f}}\left(10^{6}\right)$ & $T$ & $b$ \\
\hline Exponential & 1.31 & 1.04 & 198 & N/A \\
Growth Model & $(1.26 \sim 1.36)$ & $(1.00 \sim 1.07)$ & $(195 \sim 202)$ & \\
\hline Generalized & 1.24 & 1.26 & 213 & 1.12 \\
Growth Model & $(1.18 \sim 1.30)$ & $(1.16 \sim 1.37)$ & $(206 \sim 220)$ & $(1.07 \sim 1.15)$ \\
\hline
\end{tabular}

